\title{
Variability in innate host immune responses to cryptococcosis
}

\author{
Mariam Garelnabi, Robin C May/ ${ }^{+}$ \\ University of Birmingham, Institute of Microbiology and Infection, School of Biosciences, Edgbaston, Birmingham, UK
}

Cryptococcosis is an invasive fungal disease caused by Cryptococcus neoformans and the closely related species C. gattii. The severe form of the disease, cryptococcal meningitis (CM), is rapidly fatal without treatment. Although typically a disease of immunocompromised (especially HIV-positive) individuals, there is growing awareness of cryptococcal disease amongst non-immunocompromised patients. Whilst substantial progress has been made in understanding the pathogenicity of $C$. neoformans in HIV patients, prospective data on cryptococcosis outside the context of HIV remains lacking. Below we review how innate immune responses vary between hosts depending on immunological status, and discuss risk factors and predictors of disease outcome in different groups.

Key words: Cryptococcus neoformans - host-pathogen interactions - innate immunity - infection - pathogenesis - macrophages - host variation

Cryptococcal meningitis $(\mathrm{CM})$ remains the leading cause of fungal meningitis worldwide, afflicting up to 1 million individuals annually with approximately 600,000 subsequent deaths (Park et al. 2009). This disease arises following severe infection with members of the genus Cryptococcus in a variety of human hosts with different immune backgrounds. The majority ( $80 \%)$ of cryptococcosis cases are HIV-associated, and are caused by Cryptococcus neoformans; placing this disease as the second most common cause of AIDs-related deaths after tuberculosis (Park et al. 2009, Jarvis et al. 2010, Rajasingham et al. 2017). C. neoformans also causes CM in non-HIV, immunocompromised patients and at a rising frequency in 'otherwise healthy' individuals (Zhu et al. 2010, George et al. 2018).

Infection begins upon inhalation of cryptococcal spores from the environment, triggering the innate immune system. Macrophages play an integral role in anticryptococcal defense, with alveolar macrophages acting as first responders in the lungs where they detect and engulf cryptococcal spores (Giles et al. 2009). As intracellular pathogens, cryptococci are capable of survival and replication within host macrophages (Levitz et al. 1999, Sabiiti et al. 2014); failure to clear pulmonary infection leads to fungal dissemination throughout the body and towards the brain resulting in CM via the Trojan horse model.

Whilst early studies of the manifestations of cryptococcosis have shown clinical and prognostic differences between the infecting species (Speed and Dunt 1995), more recent investigations into the pathogenesis of $C$. neoformans in particular have revealed a critical role for host immune status in conferring protection from, or controlling disease progression towards, meningitis (Chen et al. 2000). This has led to the division of $C$. neoformans patients into three groups: HIV-associated; Non-HIV immunocompromised patients; and 'otherwise healthy' immunocompetent individuals.

doi: 10.1590/0074-02760180060

+ Corresponding author: r.c.may@bham.ac.uk

Received 1 February 2018

Accepted 2 March 2018
Hosts with intact immune systems mount an immune response that leads to clearance of the infection, or the establishment of a latent, asymptomatic infection accompanied by the formation of cryptococcomas. Patients with impaired cell-mediated immunity are unable to effectively clear C. neoformans. Thus, effective innate immune activation and a sufficient inflammatory response are key to the control of cryptococcosis. Below, we review how underlying host innate immune responses vary between the aforementioned three groups of human hosts in response to cryptococcal disease caused by C. neoformans. We also assess current understanding of how immune responses in different hosts may be predictive of protection from, or susceptibility to, CM. For a discussion of the role of adaptive immunity in C. neoformans infection, we would refer readers to the recent review by Mukaremera and Nielsen (2017).

Immune responses in non-immunocompromised individuals - During the cryptococcosis outbreak in Vancouver 2003 that affected primarily non-immunocompromised individuals, $C$. gattii was identified as a primary pathogen of the healthy. However, increased recognition of cryptococcosis cases due to $C$. neoformans in other immunocompetent patients provides strong evidence that this species also harbors capabilities as a primary pathogen, despite early classification as a strictly opportunistic pathogen.

In 'otherwise healthy' individuals, pulmonary infection is generally asymptomatic. The pattern recognition receptor (PRR) CD14 in association with Toll-like receptor 4 (TLR4), TLR2 (Shoham et al. 2001), and CD18 (Dong and Murphy 1997) on the surface of macrophages recognise the cryptococcal capsular polysaccharide glucuronoxylomannan (GXM), driving localised immune recognition and enhanced phagocytosis (Dong and Murphy 1997, Yauch et al. 2004, Giles et al. 2009, Levitz 2010, Garcia-Rodas and Zaragoza 2012). The macrophage arsenal to eliminate engulfed fungi includes the release of proinflammatory cytokines and chemokines that extend cell mediated immunity by increasing monocyte and neutrophil recruitment to the site of infection, and antigen presentation to T-cells. Secretion of these cytokines typically follows induction of the nuclear 
factor- $\kappa \mathrm{B}(\mathrm{NF}-\kappa \mathrm{B})$ pathway (Figure) that is regulated by microRNAs (miRNAs) (Chen et al. 2017) to promote the expression of inflammatory cytokines in attempts to kill the pathogen (Johnston and May 2013, Smith et al. 2015). However, this pathway can be modulated by both shed GXM and live cryptococci via different mechanisms depending on the stage of infection (Hayes et al. 2016). Early on in the infection, GXM acts as an anti-phagocytic cloak for C. neoformans (Feldmesser et al. 2000). Following ingestion by macrophages, cryptococci continue to produce and shed the polysaccharide (Feldmesser et al. 2000), which has been shown to reduce LPS-associated proinflammatory cytokine responses both in vitro (Monari et al. 2006) and in a murine infection model by inhibiting MyD88 activation following its interaction with the Fc $\gamma$ RIIB receptor (Figure) (Monari et al. 2006, Piccioni et al. 2013). Furthermore, Hayes et al. (2016) recently showed in RAW 264.7 murine macrophages that extracellular GXM inhibited LPS-induced nuclear translocation of the $\mathrm{p} 65$ protein, while intraphagosomal GXM increased the duration of nuclear translocation of the p65 and I $\kappa \mathrm{B} \alpha$ proteins, consequentially eliminating the expression of TNF $\alpha$ or inducible NOS (iNOS).

The primary defense against cryptococcosis in immunocompetent hosts is orchestrated by Th1-type CD4 ${ }^{+}$ T-cells (Kawakami et al. 1995, Wozniak et al. 2009, Olszewski et al. 2010) that produce interferon- $\gamma($ IFN- $\gamma$ ), and direct macrophages towards a Th1, classically activated, phenotype (Kawakami et al. 1995). Th1 responses have been associated with increased fungal clearance, whilst Th2 responses are associated with poor anticryptococcal defenses (Figure); thus, the interplay between Th1 and Th2 host responses during cryptococcal infection is a major determinant of disease outcome (Koguchi and Kawakami 2002, Arora et al. 2011). Host susceptibility studies in mice have shown that production of the Th1-type cytokines IFN- $\gamma$ and IL-12, as well as the pro-inflammatory cytokine TNF- $\alpha$ confers protection against cryptococcosis (Kawakami et al. 1996a, Kawakami et al. 1996b, Kawakami et al. 1996c, Decken et al. 1998, Herring et al. 2002). Conversely, production of Th2-type cytokines (including IL-4, IL-5, IL-10, and IL-13) renders hosts vulnerable to severe disease and mortality (Muller et al. 2007). GXM has also been shown to directly inhibit T-cell proliferation in mice, leading to dampened Th1 responses, and diminished resolution of the infection (Yauch et al. 2006).

Macrophage differentiation is regulated by granulocyte-macrophage colony-stimulating factor (GM-CSF). The presence of anti-GM-CSF autoantibodies in the spinal CSF inhibits the activity of GM-CSF, and has been associated with poor disease prognosis in cases of $\mathrm{CM}$ due to $C$. neoformans in otherwise healthy hosts (Rosen et al. 2013). Further attempts to characterise predispositions to $\mathrm{CM}$ have identified genetic background as a risk factor for developing severe cryptococcosis. Polymorphisms in the Fc gamma receptor (Fc $\gamma \mathrm{R}$ ) (Meletiadis et al. 2007, Hu et al. 2012) and mannose-binding lectin (MBL) (Ou et al. 2011) have been shown to increase susceptibility to cryptococcal infections in immunocompetent individuals.

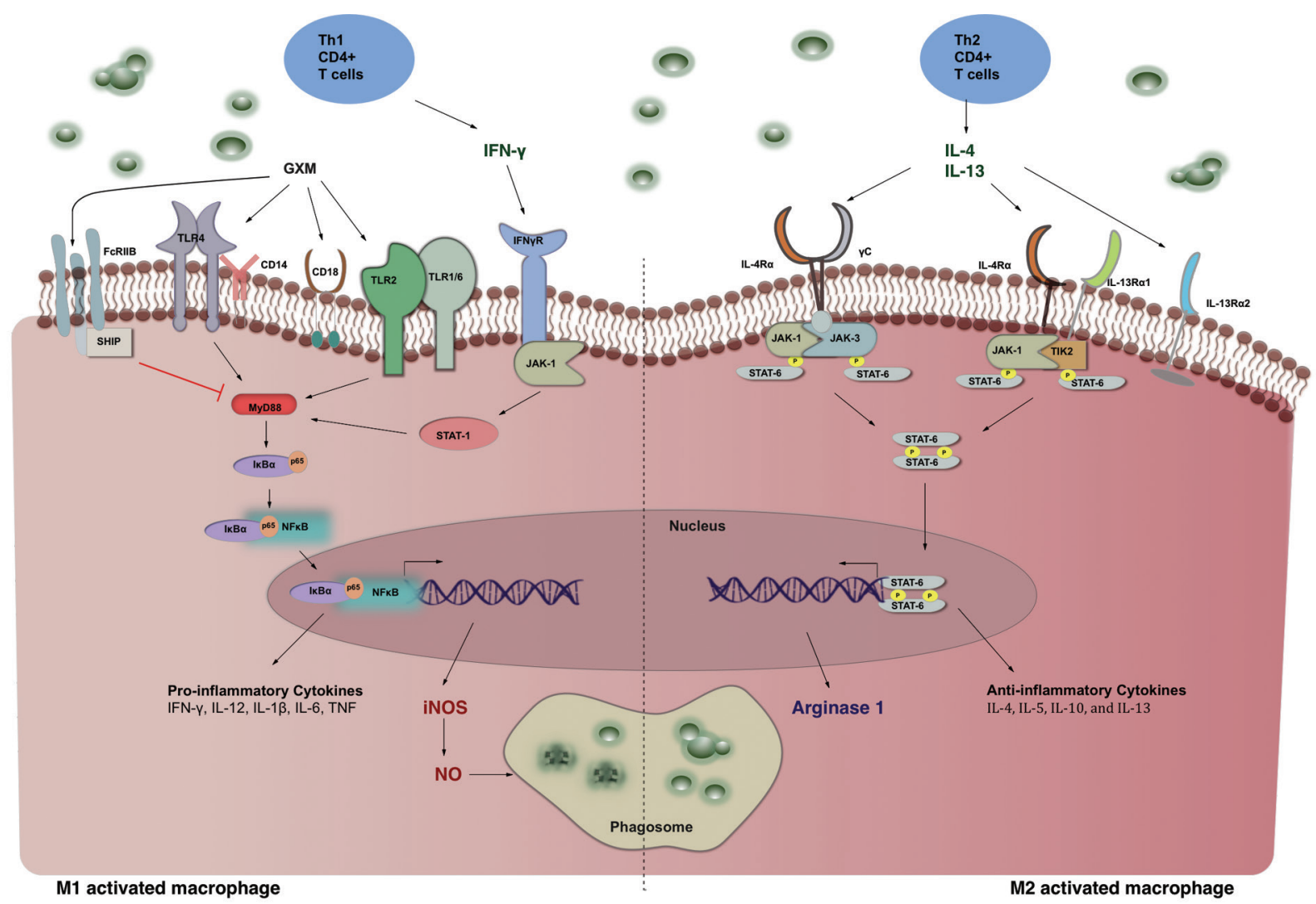

Macrophage activation state determines the outcome of Cryptococcus neoformans clearance. 
A retrospective study by Nadrous et al. (2003) of cryptococcosis in 42 immunocompetent hosts showed that $86 \%$ of patients had isolated pulmonary cryptococcosis with no evidence of fungal dissemination to the CNS, of which $33 \%$ were asymptomatic, and $92 \%$ were able to resolve the infection either with or without antifungal treatment or surgical abscission. Whilst no quantitation of innate immune responses to infection at the individual level were performed, these findings show that the majority of cryptococcosis patients with previously intact immune systems and no evidence of disseminated disease are able to effectively eliminate cryptococcal infection.

Immune responses in HIV patients - Cryptococcal meningitis is the second largest cause of mortality among HIV patients worldwide. Disease prevalence became more apparent among immunocompromised patients particularly during the HIV pandemic in the 1970s; where $80 \%$ of CM sufferers were HIV-seropositive. However, as of 2014, the frequency of cryptococcal disease among this group has dropped to a third (Mirza et al. 2003, Cox et al. 2014). Thanks to the widespread of anti-retroviral (ARV) therapies, it was estimated that 223,100 cases of $\mathrm{CM}$ in HIV patients occur each year worldwide, causing 181,100 deaths annually (Rajasingham et al. 2017).

T-cell depletion in HIV patients is a determining immunological feature that renders this group susceptible to CM. However, in a recent study, Neal et al. (2017) demonstrated in a murine model that whilst $\mathrm{CD}^{+} \mathrm{T}$-cells assist in the clearance of the fungal disease, they also contribute to disease dissemination in the CNS as well as tissue damage as a result of immunotherapy, leading to immune reconstitution inflammatory syndrome (IRIS). Reduced counts of $\mathrm{CD}^{+} \mathrm{T}$-cells in this patient group conventionally results in the proliferation of $\mathrm{CD}^{+} \mathrm{T}$-cells and increased recruitment of myeloid cell lineages to combat ongoing infection, subsequently affecting clinical outcomes of disease and deaths from CM.

Other reports have identified a number of protective immune traits and markers of susceptibility to acquiring $\mathrm{CM}$ in HIV-seropositive patients. Cerebral spinal fluid (CSF) cytokine profiles can reliably report disease progression and predict mortality in HIV patients. Data from several patient cohorts agree that inflammatory and Th1 cytokine responses confer protective advantages in HIV patients (Jarvis et al. 2014, Jarvis et al. 2015, Scriven et al. 2017). HIV-seropositive hosts with high CSF levels of interleukin (IL)-6, interferon- $\gamma$ (IFN- $\gamma$ ), IL-8, IL-12, TNF- $\alpha$ and CXCL10 show improved fungal clearance and survival rates; while increased levels of IL-4 and IL-10 have been associated with high serum levels of cryptococcal GXM that impairs monocyte activation and cripples cell-mediated responses to infection - effectively predicting mortality in this group of patients (Retini et al. 1998, Jarvis et al. 2015, Mora et al. 2015). However, low T-cell infiltration of the CSF rather than alternative macrophage activation is a major determinant of disease outcome in HIV-associated cryptococcosis (Scriven et al. 2017). Interestingly, not all HIV patients with low $\mathrm{CD}^{+} \mathrm{T}$ cell counts develop cryptococcosis. This is thought to be largely due to the contribution of genetic factors to disease susceptibil- ity. Following the association of polymorphisms in the Fc $\gamma \mathrm{R}$ with susceptibility to cryptococcosis in non-HIV patients (Meletiadis et al. 2007, Ou et al. 2011, Hu et al. 2012), Rohatgi et al. (2013) showed that HIV patients who were either heterozygous or homozygous for the FCGR3A $158 \mathrm{~V}$ allele, were 2.1- and 21-fold at higher risk of developing cryptococcal disease than individuals without the allele, respectively. The group suggested that increased recognition of $C$. neoformans and immune activation leading to a rise in phagocytic uptake as the functional implication of this allele. These findings provide an alternative approach for identification of at-risk individuals, and more personalised treatment strategies.

Immune responses in non-HIV, immunocompromised patients - This group of patients is highly heterogeneous, encompassing a range of patients from solid organ transplant (SOT) recipients to those with underlying immune defects such as sarcoidosis, diabetes mellitus, and patients receiving anti-cancer treatments (Singh et al. 2009). Similar to HIV-sufferers, organ transplant recipients also present with defects in T-cell-mediated immune responses to cryptococcosis due to immunosuppression (Pappas et al. 2001).

In SOT patients, the severity of infection has been associated with the level of immunosuppression (Husain et al. 2001). Studies by Pappas et al. (2001) have shown that the type of immunosuppressive treatment(s) administered to this group influences their susceptibility to CNS-associated cryptococcosis, responses to antifungal treatments, and likely fatality. Another study by Saha et al. (2007) provided serological evidence that the majority of cryptococcal infections in these patients were due to reactivation of pre-transplantation infections. Individuals with circulating antibodies against Cryptococcus prior to organ transplantation acquired cryptococcosis sooner than those without previous exposure to the pathogen, and were more likely to develop CM. Disease progression in this subset was also dependent on the type of organ transplanted, although transmission of infection from organ donor to recipient has rarely been observed (Singh et al. 2009).

Discussion - Whilst the reduction in global HIVassociated CM is testament to improved ART and antifungal treatment strategies, the rise in frequency of nonHIV and non-transplant associated cryptococcosis in the developed world represents a cause for concern. HIV-associated CM remains the most extensively investigated among the three groups. Whilst the majority of cryptococcal infections in previously healthy individuals are asymptomatic, clinical manifestations and prognoses vary greatly. Current clinical guidelines for this group are generated from immunocompromised cohorts and thus may not be appropriate for disease management in immunocompetent hosts. Whilst numerous case reports of 'unusual' cryptococcosis cases have been published, there is a scarcity of prospective data on the management of CM in previously healthy individuals.

A recent article by Pirofski and Casadevall (2017) discussed the effects of host-mediated damage on the progression of cryptococcosis. Given the variety of host-microbe interactions that occur within the differ- 
ent patient groups discussed above, and the associated alteration of the inflammatory immune responses, this damage-response framework (DRF) model may be particularly helpful for understanding infections in otherwise healthy individuals. Clearly, however, there is currently a lack of characterisation studies in non-HIV cryptococcal patients with which to inform this model.

It has previously been shown that cytokine expression by macrophages is not permanently biased to either Th1 or Th2 responses, and may be modulated (Arora et al. 2011); hence, the fluidity in macrophage activation states instigates varied responses in individuals with robust immune systems (Koguchi and Kawakami 2002). Furthermore, the genetic contribution to phenotypic challenges in anticryptococcal responses among cryptococcosis sufferers provides an additional tool for early diagnosis in at-risk cohorts. However, difficulties arise in the case of nonHIV immunocompetent hosts who may not be considered at-risk and therefore subject to presumptive genetic testing in comparison to their immunocompromised counterparts. In addition, it was brought to light in a recent article by Netea (2013), that the association of the above-mentioned polymorphisms with susceptibility to severe cryptococcosis is not descriptive of all patients across different cohorts depending on ethnic backgrounds.

Whilst therapeutic approaches for HIV-associated cryptococcal disease in combination with ARTs are successfully being established, efforts to determine protective features of the innate immunity in non-HIV-associated disease are still underway. Knowledge of cryptococcal pathogenesis remains minimal in patients with known immunological defects beside HIV, and treatment strategies remain unspecific. This may be at least partially due to the inappropriate clustering of these two groups together into a single "non-HIV-associated cryptococcosis" category, when in fact they represent at least two distinct cohorts. A better understanding of the sources of variation between and within patient groups is urgently needed in order to help inform strategies to appropriately modulate the immune responses at the level of the individual to improve disease outcomes.

\section{AUTHORS' CONTRIBUTION}

MG and RCM wrote the manuscript.

\section{REFERENCES}

Arora S, Olszewski MA, Tsang TM, McDonald RA, Toews GB, Huffnagle GB. Effect of cytokine interplay on macrophage polarization during chronic pulmonary infection with Cryptococcus neoformans. Infect Immun. 2011; 79(5): 1915-26.

Chen H, Jin Y, Chen H, Liao N, Wang Y, Chen J. MicroRNA-mediated inflammatory responses induced by Cryptococcus neoformans are dependent on the NF-kappaB pathway in human monocytes. Int J Mol Med. 2017; 39(6): 1525-32.

Chen S, Sorrell T, Nimmo G, Speed B, Currie B, Ellis D, et al. Epidemiology and host- and variety-dependent characteristics of infection due to Cryptococcus neoformans in Australia and New Zealand. Australasian Cryptococcal Study Group. Clin Infect Dis. 2000; 31(2): 499-508.

Cox GM, Perfect JR, Bartlett JG, Mitty J. Epidemiology, clinical manifestations, and diagnosis of Cryptococcus neoformans meningoencephalitis in HIV-infected patients. UpToDate. 2014.
Available from: http://ultra-medica.net/Uptodate21.6/contents/ mobipreview.htm?39/61/40919?source=related_link.

Decken K, Kohler G, Palmer-Lehmann K, Wunderlin A, Mattner F, Magram J, et al. Interleukin-12 is essential for a protective Th1 response in mice infected with Cryptococcus neoformans. Infect Immun. 1998; 66(10): 4994-5000.

Dong ZM, Murphy JW. Cryptococcal polysaccharides bind to CD18 on human neutrophils. Infect Immun. 1997; 65(2): 557-63.

Feldmesser M, Kress Y, Novikoff P, Casadevall A. Cryptococcus neoformans is a facultative intracellular pathogen in murine pulmonary infection. Infect Immun. 2000; 68(7): 4225-37.

Garcia-Rodas R, Zaragoza O. Catch me if you can: phagocytosis and killing avoidance by Cryptococcus neoformans. FEMS Immunol Med Microbiol. 2012; 64(2): 147-61.

George IA, Spec A, Powderly WG, Santos CAQ. Comparative epidemiology and outcomes of human immunodeficiency virus (HIV), non-HIV non-transplant, and solid organ transplant associated cryptococcosis: a population-based study. Clin Infect Dis. 2018; 66(4): 608-11.

Giles SS, Dagenais TR, Botts MR, Keller NP, Hull CM. Elucidating the pathogenesis of spores from the human fungal pathogen Cryptococcus neoformans. Infect Immun. 2009; 77(8): 3491-3500.

Hayes JB, Sircy LM, Heusinkveld LE, Ding W, Leander RN, McClelland EE, et al. Modulation of macrophage inflammatory nuclear factor kappaB (NF-kappaB) signaling by intracellular Cryptococcus neoformans. J Biol Chem. 2016; 291(30): 15614-27.

Herring AC, Lee J, McDonald RA, Toews GB, Huffnagle GB. Induction of interleukin-12 and gamma interferon requires tumor necrosis factor alpha for protective T1-cell-mediated immunity to pulmonary Cryptococcus neoformans infection. Infect Immun. 2002; 70(6): 2959-64.

Hu XP, Wu JQ, Zhu LP, Wang X, Xu B, Wang RY, et al. Association of Fcgamma receptor IIB polymorphism with cryptococcal meningitis in HIV-uninfected Chinese patients. PLoS One. 2012; 7(8): e42439.

Husain S, Wagener MM, Singh N. Cryptococcus neoformans infection in organ transplant recipients: variables influencing clinical characteristics and outcome. Emerg Infect Dis. 2001; 7(3): 375-81.

Jarvis JN, Bicanic T, Loyse A, Namarika D, Jackson A, Nussbaum JC, et al. Determinants of mortality in a combined cohort of $501 \mathrm{pa}-$ tients with HIV-associated cryptococcal meningitis: implications for improving outcomes. Clin Infect Dis. 2014; 58(5): 736-45.

Jarvis JN, Meintjes G, Bicanic T, Buffa V, Hogan L, Mo S, et al. Cerebrospinal fluid cytokine profiles predict risk of early mortality and immune reconstitution inflammatory syndrome in HIV-associated cryptococcal meningitis. PLoS Pathog. 2015; 11(4): e1004754.

Jarvis JN, Meintjes G, Williams A, Brown Y, Crede T, Harrison TS. Adult meningitis in a setting of high HIV and TB prevalence: findings from 4961 suspected cases. BMC Infect Dis. 2010; 10: 67.

Johnston SA, May RC. Cryptococcus interactions with macrophages: evasion and manipulation of the phagosome by a fungal pathogen. Cell Microbiol. 2013; 15(3): 403-11.

Kawakami K, Kohno S, Kadota J, Tohyama M, Teruya K, Kudeken N, et al. $\mathrm{T}$ cell-dependent activation of macrophages and enhancement of their phagocytic activity in the lungs of mice inoculated with heat-killed Cryptococcus neoformans: involvement of IFNgamma and its protective effect against cryptococcal infection. Microbiol Immunol. 1995; 39(2): 135-43.

Kawakami K, Qifeng X, Tohyama M, Qureshi MH, Saito A. Contribution of tumour necrosis factor-alpha (TNF-alpha) in host defence mechanism against Cryptococcus neoformans. Clin Exp Immunol. 1996a; 106(3): 468-74. 
Kawakami K, Tohyama M, Teruya K, Kudeken N, Xie Q, Saito A. Contribution of interferon-gamma in protecting mice during pulmonary and disseminated infection with Cryptococcus neoformans. FEMS Immunol Med Microbiol. 1996b; 13(2): 123-30.

Kawakami K, Tohyama M, Xie Q, Saito A. IL-12 protects mice against pulmonary and disseminated infection caused by Cryptococcus neoformans. Clin Exp Immunol. 1996c; 104(2): 208-14.

Koguchi Y, Kawakami K. Cryptococcal infection and Th1-Th2 cytokine balance. Int Rev Immunol. 2002; 21(4-5): 423-38.

Levitz SM, Nong SH, Seetoo KF, Harrison TS, Speizer RA, Simons ER. Cryptococcus neoformans resides in an acidic phagolysosome of human macrophages. Infect Immun. 1999; 67(2): 885-90.

Levitz SM. Innate recognition of fungal cell walls. PLoS Pathog. 2010; 6(4): e1000758.

Meletiadis J, Walsh TJ, Choi EH, Pappas PG, Ennis D, Douglas J, et al. Study of common functional genetic polymorphisms of FCGR2A, 3A and 3B genes and the risk for cryptococcosis in HIVuninfected patients. Medical Mycology. 2007; 45(6): 513-8.

Mirza SA, Phelan M, Rimland D, Graviss E, Hamill R, Brandt ME, et al. The changing epidemiology of cryptococcosis: an update from population-based active surveillance in 2 large metropolitan areas, 1992-2000. Clin Infect Dis. 2003; 36(6): 789-94.

Monari C, Kozel TR, Paganelli F, Pericolini E, Perito S, Bistoni F, et al. Microbial immune suppression mediated by direct engagement of inhibitory Fc receptor. J Immunol. 2006; 177(10): 6842-51.

Mora DJ, Fortunato LR, Andrade-Silva LE, Ferreira-Paim K, Rocha $\mathrm{IH}$, Vasconcelos RR, et al. Cytokine profiles at admission can be related to outcome in AIDS patients with cryptococcal meningitis. PLoS One. 2015; 10(3): e0120297.

Mukaremera L, Nielsen K. Adaptive immunity to Cryptococcus neoformans infections. J Fungi (Basel). 2017; 3(4): pi:64.

Muller U, Stenzel W, Kohler G, Werner C, Polte T, Hansen G, et al. IL-13 induces disease-promoting type 2 cytokines, alternatively activated macrophages and allergic inflammation during pulmonary infection of mice with Cryptococcus neoformans. J Immunol. 2007; 179(8): 5367-77.

Nadrous HF, Antonios VS, Terrell CL, Ryu JH. Pulmonary cryptococcosis in nonimmunocompromised patients. Chest. 2003; 124(6): 2143-7.

Neal LM, Xing E, Xu J, Kolbe JL, Osterholzer JJ, Segal BM, et al. $\mathrm{CD} 4(+) \mathrm{T}$ cells orchestrate lethal immune pathology despite fungal clearance during Cryptococcus neoformans meningoencephalitis. MBio. 2017; 8(6): e01415-17.

Netea MG. Toward identification of the genetic risk profile for cryptococcal disease in HIV-infected patients. MBio. 2013; 4(5): e00798-00713.

Olszewski MA, Zhang Y, Huffnagle GB. Mechanisms of cryptococcal virulence and persistence. Future Microbiol. 2010; 5(8): 1269-88.

Ou XT, Wu JQ, Zhu LP, Guan M, Xu B, Hu XP, et al. Genotypes coding for mannose-binding lectin deficiency correlated with cryptococcal meningitis in HIV-uninfected Chinese patients. J Infect Dis. 2011; 203(11): 1686-91.

Pappas PG, Perfect JR, Cloud GA, Larsen RA, Pankey GA, Lancaster DJ, et al. Cryptococcosis in human immunodeficiency virusnegative patients in the era of effective azole therapy. Clin Infect Dis. 2001; 33(5): 690-9.

Park BJ, Wannemuehler KA, Marston BJ, Govender N, Pappas PG, Chiller TM. Estimation of the current global burden of cryptococcal meningitis among persons living with HIV/AIDS. Aids. 2009; 23(4): 525-30
Piccioni M, Monari C, Kenno S, Pericolini E, Gabrielli E, Pietrella $\mathrm{D}$, et al. A purified capsular polysaccharide markedly inhibits inflammatory response during endotoxic shock. Infect Immun. 2013; 81(1): 90-8.

Pirofski LA, Casadevall A. Immune-mediated damage completes the parabola: Cryptococcus neoformans pathogenesis can reflect the outcome of a weak or strong immune response. MBio. 2017; 8(6): e02063-17.

Rajasingham R, Smith RM, Park BJ, Jarvis JN, Govender NP, Chiller $\mathrm{TM}$, et al. Global burden of disease of HIV-associated cryptococcal meningitis: an updated analysis. Lancet Infect Dis. 2017; 17(8): 873-81.

Retini C, Vecchiarelli A, Monari C, Bistoni F, Kozel TR. Encapsulation of Cryptococcus neoformans with glucuronoxylomannan inhibits the antigen-presenting capacity of monocytes. Infect Immun. 1998; 66(2): 664-9.

Rohatgi S, Gohil S, Kuniholm MH, Schultz H, Dufaud C, Armour $\mathrm{KL}$, et al. Fc gamma receptor $3 \mathrm{~A}$ polymorphism and risk for HIVassociated cryptococcal disease. MBio. 2013; 4(5): e00573-00513.

Rosen LB, Freeman AF, Yang LM, Jutivorakool K, Olivier KN, Angkasekwinai N, et al. Anti-GM-CSF autoantibodies in patients with cryptococcal meningitis. J Immunol. 2013; 190(8): 3959-66.

Sabiiti W, Robertson E, Beale MA, Johnston SA, Brouwer AE, Loyse A, et al. Efficient phagocytosis and laccase activity affect the outcome of HIV-associated cryptococcosis. J Clin Invest. 2014; 124(5): 2000-8.

Saha DC, Goldman DL, Shao X, Casadevall A, Husain S, Limaye AP, et al. Serologic evidence for reactivation of cryptococcosis in solid-organ transplant recipients. Clin Vaccine Immunol. 2007; 14(12): 1550-4.

Scriven JE, Graham LM, Schutz C, Scriba TJ, Wilkinson KA, Wilkinson RJ, et al. The CSF immune response in HIV-1-associated cryptococcal meningitis: macrophage activation, correlates of disease severity, and effect of antiretroviral therapy. J Acquir Immune Defic Syndr. 2017; 75(3): 299-307.

Shoham S, Huang C, Chen JM, Golenbock DT, Levitz SM. Toll-like receptor 4 mediates intracellular signaling without TNF-alpha release in response to Cryptococcus neoformans polysaccharide capsule. J Immunol. 2001; 166(7): 4620-6.

Singh N, Forrest G, AST Infectious Diseases Community of Practice. Cryptococcosis in solid organ transplant recipients. Am J Transplant. 2009; 9(Suppl 4): S192-8.

Smith LM, Dixon EF, May RC. The fungal pathogen Cryptococcus neoformans manipulates macrophage phagosome maturation. Cell Microbiol. 2015; 17(5): 702-13.

Speed B, Dunt D. Clinical and host differences between infections with the two varieties of Cryptococcus neoformans. Clin Infect Dis. 1995; 21(1): 28-34.

Wozniak KL, Ravi S, Macias S, Young ML, Olszewski MA, Steele $\mathrm{C}$, et al. Insights into the mechanisms of protective immunity against Cryptococcus neoformans infection using a mouse model of pulmonary cryptococcosis. PLoS One. 2009; 4(9): e6854.

Yauch LE, Lam JS, Levitz SM. Direct inhibition of T-cell responses by the Cryptococcus capsular polysaccharide glucuronoxylomannan. PLoS Pathog. 2006; 2(11): e120.

Yauch LE, Mansour MK, Shoham S, Rottman JB, Levitz SM. Involvement of CD14, Toll-Like receptors 2 and 4, and MyD88 in the host response to the fungal pathogen Cryptococcus neoformans in vivo. Infect Immun. 2004; 72(9): 5373-82.

Zhu LP, Wu JQ, Xu B, Ou XT, Zhang QQ, Weng XH. Cryptococcal meningitis in non-HIV-infected patients in a Chinese tertiary care hospital, 1997-2007. Med Mycol. 2010; 48(4): 570-9. 